\title{
ONERA-DLR bistatic SAR campaign: planning, data acquisition, and first analysis of bistatic scattering behaviour of natural and urban targets
}

\author{
P. Dubois-Fernandez, H. Cantalloube, B. Vaizan, G. Krieger, R. Horn, M. Wendler and V. Giroux
}

\begin{abstract}
Bistatic configuration is an attractive concept for spaceborne and airborne SAR missions when distributed radars are necessary as for example in the case of interferometric applications. The first reason is the important cost reduction achieved over the multiple radar elements, by having only one transmitter (expensive part) and multiple receivers. The most promising applications are single-pass interferometry with a large baseline and target or surface characterisation from bistatic scattering signature analysis. In a defence context, the improved stealth associated with the receive-only component can provide a wider operational capability. In order to explore the potentials and technical challenges associated with bistatic radar, DLR and ONERA have conducted a joint bistatic airborne radar experiment involving both their SAR systems E-SAR and RAMSES between October 2002 and February 2003. Two main geometrical configurations were flown to explore different scientific and technical objectives. In the first geometrical configuration, the quasi-monostatic mode, the two planes were flying very close to each other to acquire interferometric data in a single-pass cross-platform configuration with large interferometric baselines. The second geometrical configuration was designed to acquire images with a large bistatic angle. The two planes were flying on parallel tracks around $2 \mathrm{~km}$ apart, at about the same altitude, with the antennas pointing at the same area on the ground. The authors describe this research programme, including the preparation phase, the analysis of the technological challenges that had to be solved before the acquisition, the strategy adopted for bistatic image processing, the first results and a preliminary analysis of the acquired images.
\end{abstract}

\section{Introduction}

Bistatic synthetic aperture radar enables a wealth of new and powerful imaging modes [1-3]. One prominent example is spaceborne cross-track interferometry employing distinct platforms [4-6]. The scientific applications linked to interferometry are spectacular and have generated a lot of interest [7, 8]. However, classical spaceborne repeat pass interferometry suffers from two main difficulties, namely atmospheric disturbances and temporal decorrelation. Acquiring single pass interferometric data is attractive since these two sources of distortion disappear. However, the distance between the two antennas needs to be sufficient to provide a satisfactory height resolution in case of DEM generation. The X-SAR SRTM mission [9] on board the space shuttle Endeavour had a $60 \mathrm{~m}$-long mast to provide for this antenna separation. This option is not realistic for the typical spaceborne SAR altitude where an equivalent mast (for the same height resolution) would have to be

(C) The Institution of Engineering and Technology 2006

IEE Proceedings online no. 20045117

doi:10.1049/ip-rsn:20045117

Paper first received 27th October 2004 and in revised form 30th March 2005

P. Dubois-Fernandez, H. Cantalloube, B. Vaizan and V. Giroux are with Office National d'Etude et Recherches Aérospatiales, Departement Electromagnetisme et Radar, Salon Air Cedex BA701 13661, France

G. Krieger, R. Horn and M. Wendler are with DLR, Institute of Radio Frequency Technology and Radar Systems, P.O. Box 1116, 82230 Wessling, Germany

E-mail: pdubois@onera.fr more than twice as long. Putting two radar systems in orbit on two different platforms is then the only option. A less costly alternative to two fully active radar systems is to have only one transmitter in conjunction with two or more passive receivers, concentrating the active part on one platform as it is the major cost contributor $[5,6]$. Such a bistatic constellation is attractive but raises a complete range of new technological challenges.

A lot of attention has also been given to a concept where one radar in space illuminates a large portion of the Earth and the receiving radars are either on airplanes or low orbiting satellites acquiring images on demand [10]. The costly part of the radar is then shared among many users.

In order to explore the challenges associated with bistatic radar, DLR and ONERA organised a joint bistatic airborne experiment involving their radar systems E-SAR and RAMSES, respectively. This experiment took place between October 2002, when the first joint tests with both systems were conducted, and February 2003 when the planes were flown over Provence in the South of France. At around the same time, two other bistatic experiments were conducted in Europe [11, 12] indicating the high level of interest raised by the subject. We published our first results in the ASAR2003 Proceedings, Montréal, Canada [13] and at the International Radar Symposium in Dresden, Germany [14].

Two main geometrical configurations were flown to explore different scientific and technical objectives:

- In the first geometrical configuration, the quasi-monostatic mode, the two planes were flying very close to each other, 
one following the other, to investigate phase synchronisation and to simulate single pass cross-platform interferometry from space with a large baseline. The across-track distance was selected such that it was well below the critical baseline in order to ensure sufficient coherence between the monostatic and bistatic data sets.

- The second geometrical configuration was designed to acquire images with a large bistatic angle. The two planes were flying on parallel tracks around $2 \mathrm{~km}$ apart, at about the same altitude, with the antennas pointing at the same area on the ground.

In the first part of this paper, we describe the campaign objectives, the pre-flight engineering phase when tests were conducted to understand how to operate both radars in a bistatic mode and when an analysis was done to define the flight configurations. The second part of the paper describes the data acquisition plan and provides a short description of the preliminary processing chain that has been developed at ONERA. The third part of the paper describes the radiometric calibration procedure used for this data set and presents the images acquired with different bistatic angles together with a discussion on the typical bistatic scattering behaviour of natural surfaces.

\section{Planning the experiment}

When the campaign was first decided, it was understood that it had to be done without major system upgrades and at minimum cost. We started to investigate the compatibility of the two SARs. In the following paragraphs, the main characteristics of the two systems are given.

\subsection{RAMSES radar system}

RAMSES is a radar imaging system flown onboard a Transall C-160 aircraft operated by the French CEV (Centre d'Essais en Vol). It can be best described as an experimental test bench for radar imaging with a high modularity and flexibility [15]. For each acquisition campaign, it can be configured with three bands picked among eight possible choices ranging from P-band to W-band. Two selected frequencies can then be operated simultaneously. Once the system is mounted on the aircraft, the radar acquisition configuration can be varied from pass to pass. Six of the bands (all except $\mathrm{Ka}$ and $\mathrm{W}$ ) can be operated from a single polarisation mode to a fully polarimetric mode. The associated bandwidth and waveforms can be adjusted to best meet the data acquisition objectives (optimising swath-width against range resolution for example) and the antenna boresight incidence angle can be set from $30^{\circ}$ to $85^{\circ}$. Some bands are available with $1.2 \mathrm{GHz}$ bandwidth. The $\mathrm{X}$ band and the $\mathrm{Ku}$ band systems are interferometric [15] and can be flown in a 'polarimetric interferometry' mode. Table 1 describes the system.

\subsection{E-SAR system}

E-SAR, the airborne experimental SAR system of DLR, has been designed originally as a testbed for new radar technologies and processing algorithms and has been upgraded and expanded over the years to a multi-channel SAR system with high flexibility and innovative operating modes [16]. The radar is operational in P-, L-, C- and X-band with selectable vertical or horizontal antenna polarisations. In $\mathrm{P}$ - and L-band the system can be operated fully polarimetrically. Interferometric data acquisition is possible in X-band in single-pass and in L- and P-band in repeat-pass mode.
Table 1: Main characteristics of RAMSES system

\begin{tabular}{llclll}
\hline Band & $\begin{array}{l}\text { Centre } \\
\text { freq. }(\mathrm{GHz})\end{array}$ & $\begin{array}{l}\text { Bandwidth } \\
\text { (MHz) }\end{array}$ & Anten. & Polar. & Mode \\
\hline $\mathrm{P}$ & 0.43 & 75 & Array & Full & \\
$\mathrm{L}$ & 1.3 & 200 & Array & Full & \\
$\mathrm{S}$ & 3.2 & 300 & Array & Full & \\
$\mathrm{C}$ & 5.3 & 300 & Array & Full & \\
$\mathrm{X}$ & 9.5 & 1200 & Both & Full & PollnSAR, \\
& & & & & Multi-B \\
Ku & 14.3 & 1200 & Horn & Full & PollnSAR, \\
& & & & & Multi-B \\
Ka & 35 & 1200 & Horn & VV, HH & \\
W & 95 & 500 & Horn & LR, LL & \\
\hline
\end{tabular}

RAMSES is always right looking. PollnSAR indicates that the system can be operated in a single-pass polarimetric interferometric mode and Multi-B stands for single-pass multibaseline (at least 3 antennas). The letters in the polarisation column indicate the polarisation of the transmit antenna (first letter) and of the receive antenna (second letter) with $V$ for vertical, $\mathrm{H}$ for horizontal, $\mathrm{L}$ for left circular and $\mathrm{R}$ for right circular

The resolution of the E-SAR image products is up to $1.5 \mathrm{~m}$ in slant range and $0.5 \mathrm{~m}$ in azimuth (single look). Typical swath widths are 3 and $5 \mathrm{~km}$ and the scene length is not limited in general. The precision navigation system onboard the Dornier 228-212 aircraft assures the measurement of the platform position with an accuracy of $0.1 \mathrm{~m}$ absolute, and of its attitude by 0.01 degrees for pitch and roll and 0.1 degrees for yaw angle. The system also gives the pilot an online control about the actual flight path to help keeping the nominal track with an accuracy better than $3 \mathrm{~m}$. Table 2 describes the E-SAR system.

\subsection{Selection of the frequency}

After the first discussions, $\mathrm{X}$ - and L-bands were identified as the two possible candidates for this experiment. RAMSES is always pointing its antennas to the right through the side door of the Transall. E-SAR has its antennas attached directly on the fuselage and depending on the frequency and system, the radar is right or leftlooking. As a first experiment, in order to answer the scientific and technical objectives highlighted above, it was decided to start the investigation with the bistatic configurations were both radars are looking in the same direction. The X-band with the interferometric antenna for E-SAR was then the only possibility. RAMSES has three different X-band antennas with different beam shapes adapted to different applications. The horn antenna, characterised by the widest beam width, was selected as a conservative choice in order to lower the requirements on relative position and attitude accuracy between the airplanes as required for an overlap of the RAMSES and E-SAR antenna beam patterns.

The centre frequencies of the two X-band systems (RAMSES and E-SAR) are offset by $140 \mathrm{MHz}$. RAMSES was able to slightly shift its frequency to adapt to the E-SAR one. The E-SAR maximum chirp bandwidth is $100 \mathrm{MHz}$, and it was decided to use the $100 \mathrm{MHz}$ and also a $50 \mathrm{MHz}$ mode allowing a lower sampling frequency and as a direct consequence a longer recording window as both systems are datarate constrained. The longer recording window enabled a recording of the direct air-to-air transmit 
Table 2: Main characteristics of the E-SAR system

\begin{tabular}{lllllll}
\hline Band & Centre freq. $(\mathrm{GHz})$ & Bandwidth $(\mathrm{MHz})$ & Anten. & Polar. & Mode & Look direction \\
\hline $\mathrm{P}$ & 0.35 & 100 & Array & Full & & Left looking \\
$\mathrm{L}$ & 1.3 & 100 & Array & Full & & Left looking \\
$\mathrm{C}$ & 5.3 & 100 & Array & $\mathrm{H} \mathrm{\&} \mathrm{V}$ & $\mathrm{VH} / \mathrm{VV}$ or HV/HH & Left looking \\
$\mathrm{X}$ & 9.6 & 100 & Horn & VV or HH & single pol & Left looking \\
& & & Array & VV & XTI or ATI & Right looking \\
\hline
\end{tabular}

Letters in the polarisation column indicate the polarisation of the transmit antenna (first letter) and of the receive antenna (second letter) with $\mathrm{V}$ for vertical, $\mathrm{H}$ for horizontal, $\mathrm{L}$ for left circular and $\mathrm{R}$ for right circular

pulses and provides a useful margin in case the two pulse repetition frequencies (PRF) get desynchronised.

In Table 3, a more detailed description of both X-band systems is given. It is interesting to note the higher transmit power associated with E-SAR, resulting from a higher operational altitude. The E-SAR boresight incidence angle is set to $55^{\circ}$ which for the nominal monostatic mode of operation of the DLR system is perfectly adapted as the usual data set has a swath wide enough to explore a sufficient range of incidence angles. RAMSES can vary its boresight incidence angle from $30^{\circ}$ to $85^{\circ}$. All these characteristics have a definite impact on the bistatic configuration definition and a careful analysis of the different potential configurations had to be conducted.

\subsection{Defining the configuration}

Two types of configuration were explored. These configurations are shown in Fig. 1. In the 'ONERA' configurations, the influence of the bistatic angle is explored by flying the two planes on parallel tracks with about a $2 \mathrm{~km}$ acrosstrack offset. In the ONERA-grazing configuration, the E-SAR boresight incidence angle (BIA) is $55^{\circ}$ and the RAMSES BIA is $75^{\circ}$, creating a bistatic angle of around $20^{\circ}$. In the other ONERA configuration, RAMSES was flying on the right side of E-SAR with a BIA of $30^{\circ}$.

The DLR configurations were designed to study bistatic synchronisation and cross-platform interferometry, and the two planes were flown on almost the same track, RAMSES following closely E-SAR, with a slightly lower altitude. The distance between the two planes was around $30 \mathrm{~m}$.

The geometry associated with each configuration was fine-tuned to optimise the SAR performances, e.g., the signal-to-noise ratio (SNR), the range resolution, the timing issues $[17,18]$. In the timing analysis, we simply made sure that both the direct path (from one plane to the other) and the specular path (plane 1 - ground forward

Table 3: RAMSES and E-SAR X-band systems

\begin{tabular}{lll}
\hline & RAMSES & E-SAR \\
\hline Centre frequency (GHz) & 9.46 & 9.6 \\
Maximum chirp bandwidth & 600 & 100 \\
Polarisation & Quad pol & $\mathrm{VV}$ \\
Azimuth beamwidth & $16^{\circ}$ & $8^{\circ}$ \\
Elevation beamwidth & $16^{\circ}$ & $35^{\circ}$ \\
Boresight incidence angle & {$\left[30^{\circ}-75^{\circ}\right]$} & $55^{\circ}$ \\
Antenna look direction & Right & Right \\
Bits per sample & 8 & 6 \\
Peak transmit power $(\mathrm{W})$ & 300 & 1000 \\
\hline
\end{tabular}

scattering - plane 2) did not interfere with the useful data as described in Fig. 2. In Fig. 3, the variation of the signal-to-noise ratio is presented for the DLR configuration. It can be observed that the two antenna footprint centres are not co-located. This is a deliberate choice to provide for the best possible composite bistatic (round-trip) 'antenna pattern' shown as a red dotted line in the Figure.

One peculiar characteristic of the bistatic configurations is clearly illustrated in Fig. 3. In monostatic processing, the area within the $3 \mathrm{~dB}$ antenna pattern is used. These zones are indicated as solid blue and red lines. The antenna pattern is usually very well known in these areas.
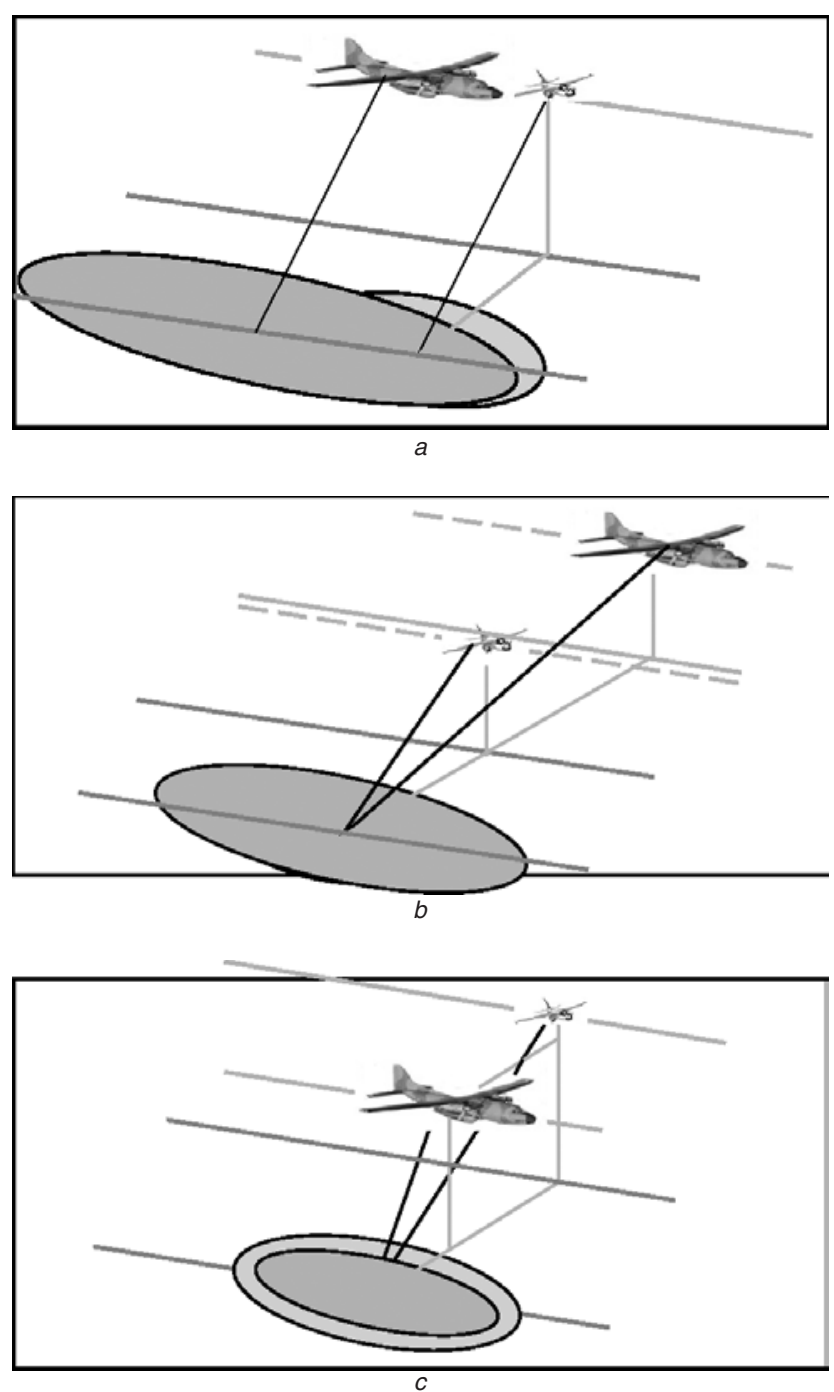

Fig. 1 Bistatic configuration

$a$ Quasi-monostatic case (BIA E-SAR $55^{\circ}$, RAMSES $45^{\circ}$ )

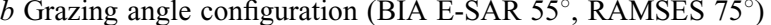
$c$ Steep angle configuration (BIA E-SAR $55^{\circ}$, RAMSES $30^{\circ}$ ) 


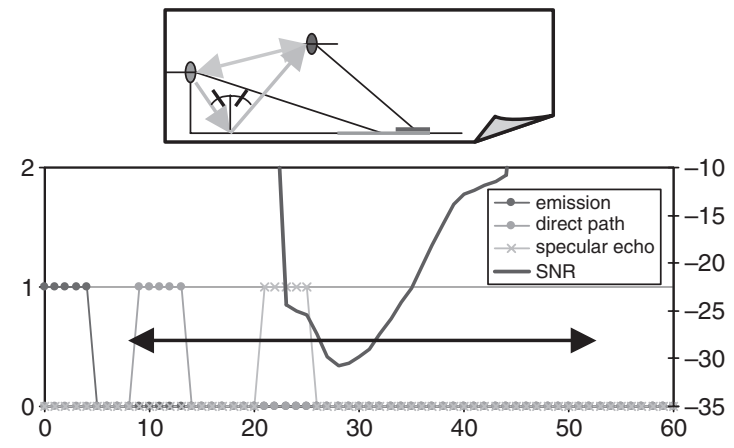

Fig. 2 Characteristic signals for timing analysis

However, as outlined by the dotted line, the corresponding $3 \mathrm{~dB}$ pattern in bistatic mode includes zones outside the $3 \mathrm{~dB}$ pattern of either E-SAR or RAMSES. The pattern is not as well-characterised there and this will create difficulties in the calibration process.

In the ONERA bistatic configurations, the two aircraft altitudes could have been adjusted such that the intersection of the antenna boresight and the ground would match However, this option was not taken for three reasons:

- SNR issues

- RAMSES flight altitude is limited to 12000 feet because we operate with an open door

- Specific request from the pilots to fly the two systems at a similar altitude in order to facilitate the control of the relative positions of the two airplanes.

The finally selected geometrical configurations have the following characteristics, shown in Table 4.

Several technological challenges had to be overcome for the acquisition. The main one was the synchronisation between the two radar systems. The second issue concerned the relative geometry control strategy.

\subsection{Pre-flight testing, trying to solve for the synchronisation issues}

The two radar systems have their own stable oscillators and if the stability of the clock during one acquisition is of major concern for SAR operation, the exact central frequency of this clock is not essential in monostatic operation: a shift of a few hertz will not affect the data quality as the same clock is used in the transmit and receive channels. However, in bistatic operation, this becomes a major issue $[1,19,20]$. This is especially the case for our experiment where the two radars had no communication link to exchange timing information. Two major difficulties can be identified: a slightly different central frequency for the transmit signal or for the sampling frequency and the positioning of the receiving window on the passive system. Both points are developed in the following paragraphs.

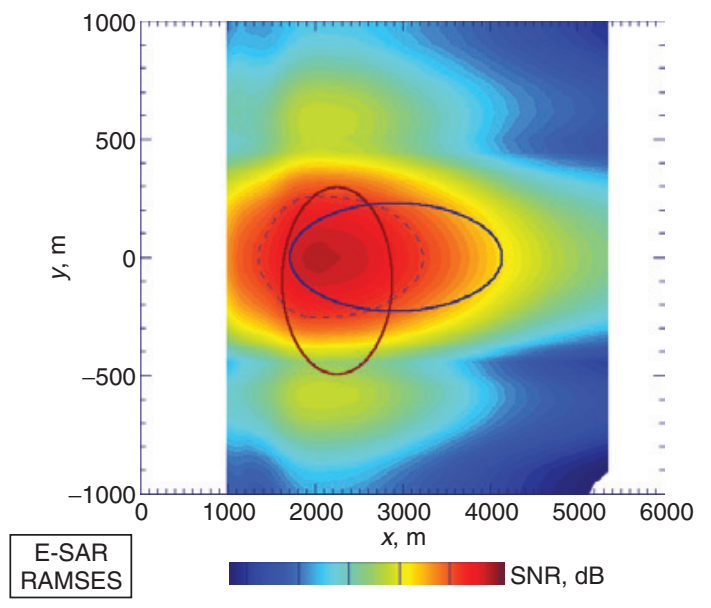

Fig. $3 S N R$ variation in the quasi-monostatic configuration where the blue and red lines are respectively the E-SAR and RAMSES antenna footprints

Red dash line is the $3 \mathrm{~dB}$ bistatic pattern and it is clearly out of the $3 \mathrm{~dB}$ ONERA and E-SAR zone

The acquisition systems of E-SAR and RAMSES cannot operate in a continuous sampling and recording mode. Therefore, for each pulse, there is only a limited window during which the signal is digitised and recorded. This window has to be positioned properly and in the monostatic case, it is done with respect to the pulse repetition frequency (PRF) signal as this signal also triggers the transmit pulse. In the bistatic case, the PRF signal on the passive system (triggering the timing of the system) may occur at a different time as the PRF signal on the active system. Both RAMSES and E-SAR operate with a $10 \mathrm{MHz}$ STALO (stable oscillator) frequency. Based on the configurations we proposed earlier and a pulse length of around $5 \mu \mathrm{s}$, a useful recording window of the order of $16 \mu \mathrm{s}$ is typical. For a PRF of $1 \mathrm{kHz}$ and a sampling rate of $100 \mathrm{MHz}$, the maximum recording window size E-SAR can have in this mode is of the order of $25 \mu \mathrm{s}$, giving us a $4.5 \mu \mathrm{s}$ time margin at the beginning and end of the recording window. Both systems are using the same type of STALO. These STALOs are extremely stable but the inherent relative drift, even if very small, can create an unacceptable shift in the recording window. The flights were planned to last around 3 hours. For a 3 hour flight, this translates into a relative maximum shift between the two STALO frequencies $\left(\Delta f / f \leq 10^{-9}\right)$. The resulting frequency stability requires a STALO of the class of a rubidium clock, known however to be sensitive to vibration and to have poor phase noise characteristics. Such an upgrade was too costly. Another solution had to be found.

The two systems include a GPS receiver with a $10 \mathrm{MHz}$ internal clock, controlled by the pulse per second (PPS) signal. These clocks are therefore available on both radars. Characterisation of the associated performances of a SAR system mastered by such a clock was done in the

Table 4: Geometry of flight configurations

\begin{tabular}{|c|c|c|c|c|c|c|}
\hline Config. ID & $\begin{array}{l}\text { Dornier } \\
\text { altitude (ft) }\end{array}$ & $\begin{array}{l}\text { Transall } \\
\text { altitude (ft) }\end{array}$ & $\begin{array}{l}\text { Horizontal cross-track } \\
\text { distance }(\mathrm{m})\end{array}$ & $\begin{array}{l}\text { Vertical cross-track } \\
\text { distance }(\mathrm{m})\end{array}$ & $\begin{array}{l}\text { Along-track } \\
\text { distance }(\mathrm{m})\end{array}$ & $\begin{array}{l}\text { Transall } \\
\text { BIA }\left(^{\circ}\right)\end{array}$ \\
\hline DLR IF 1 & 5900 & 5850 & 0 & 20 & $<100$ & 45 \\
\hline DLR IF 3 & 11500 & 11450 & 0 & 20 & $<100$ & 45 \\
\hline ONERA 1 & 9500 & 10000 & 2900 & 150 & 0 & 30 \\
\hline ONERA 2 & 3500 & 3000 & 2400 & 150 & 0 & 75 \\
\hline
\end{tabular}


laboratory. Because these clocks are constantly adjusted to match the PPS signal information, their actual frequencies are constantly changing creating an unacceptable phase noise in the radar signal if used as a STALO.

An intermediate solution was engineered. Both radars are controlled by their own internal STALO, avoiding the phase noise effect. Before take-off, the two STALOs are connected and their frequencies matched as precisely as possible. Once disconnected, the two STALOs are left undisturbed. Then, just before each acquisition, the two PRF signals are synchronised to the PPS coming from the GPS system. This insures that the recording window on the passive system is set properly to record useful data even if the frequencies of the two STALO are not exactly matched or are slowly drifting during the acquisition. This discrepancy between the two STALO (shift and/or drift) can be observed in the resulting data and has to be corrected for in the data processing chain.

\subsection{Navigation issues}

The overlapping of the two antenna footprints on the ground is an essential condition for data quality: as a consequence, the two planes have to be flown precisely together in order to maintain the proper relative geometry during each data acquisition. The two types of configuration are quite different. In the DLR case, where the two planes are flying one behind the other in a very compact formation, the Transall pilot was simply following the Dornier plane as closely as possible, in a manoeuvre quite familiar to military pilots as it resembles the in-flight refuelling phase of fighter aircraft.

The ONERA configuration is more challenging as the two planes were about $2.5 \mathrm{~km}$ apart. An analysis of the relative shift of the antenna footprints owing to positioning and attitude errors between the two planes indicated that a maximum along-track displacement of $100 \mathrm{~m}$ is allowed as described in Fig. 4. A similar analysis was done on the roll, squint and pitch angles. A $100 \mathrm{~m}$ positioning requirement over a $2.5 \mathrm{~km}$ distance cannot be met with visual means only. An alignment procedure was defined involving two predefined sets of ground control points, one for each aircraft. The corresponding points had to be overflown simultaneously. The pilots adjusted their speed to a known airspeed velocity and had six nautical miles before acquisition to line up the two planes using the ground points and by communicating through VHF channels.

This alignment phase was also used to synchronise both PRF signals by resetting the internal PRF counter in each radar system to the GPS PPS signal.
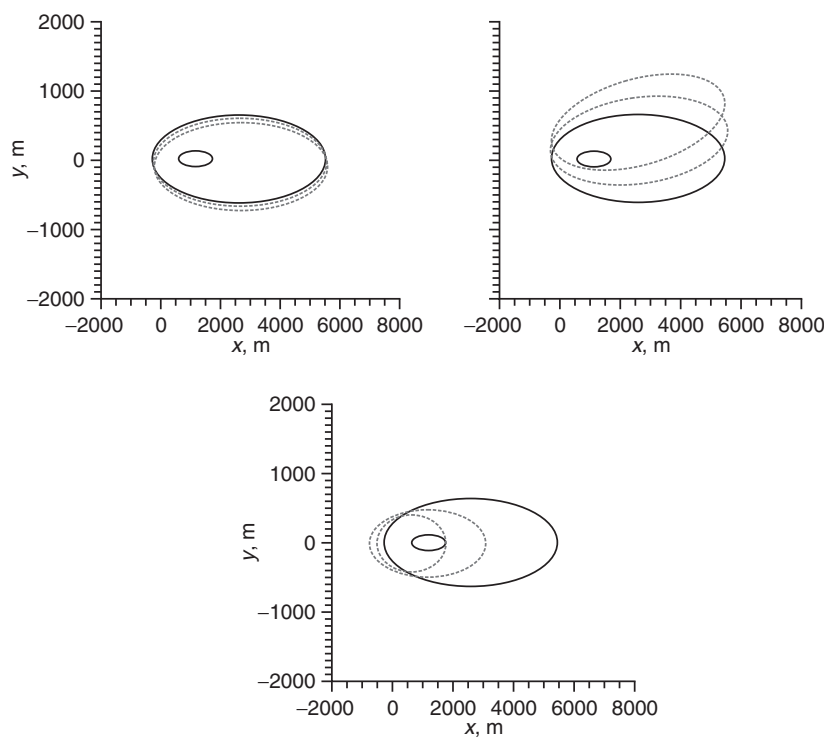

Fig. 4 Analysis of the effect of positioning errors (along track, squint and roll angles) on the antenna footprint overlap for the grazing configuration

E-SAR/RAMSES $3 \mathrm{~dB}$ antenna patterns are presented respectively by large and small footprints. The first plot corresponds to an along track error, the second to a squint error and the third to a roll angle error

\section{The campaign}

\subsection{Description of the campaign}

In all acquisitions, both systems are receiving simultaneously so that a monostatic image is always acquired for each bistatic image. For each geometrical configuration, several data takes were acquired with varying system parameters (bandwidth of $50 \mathrm{MHz}$ or $100 \mathrm{MHz}$, RAMSES transmitting or E-SAR transmitting). The campaign was composed of two flights. On the first flight, RAMSES was receiving in a dual-polarisation mode. During the second flight, RAMSES was receiving on two interferometric antennas.

During the experiment, two trihedral corner reflectors and one Luneberg sphere were deployed to calibrate the monostatic images. The calibration targets are presented in Fig. 5. The DLR configuration is fairly close to the monostatic case as the two planes are flying very close to each other. This is referred to as a quasi-monostatic mode as the bistatic angle is smaller than $0.1^{\circ}$. Both monostatic and quasi-monostatic images can be radiometrically calibrated using the passive targets. The ONERA configurations however have a large bistatic angle and this calibration option is not possible. In fact a trihedral reflector has a wide monostatic beam pattern but a very narrow bistatic pattern as shown in Fig. 6 .

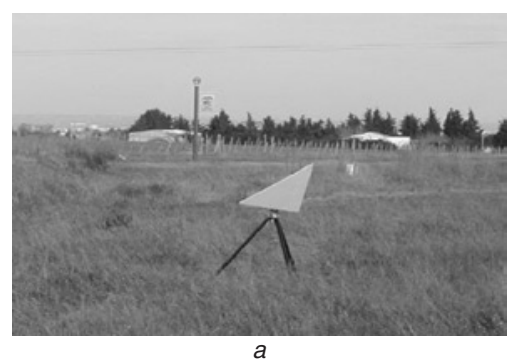

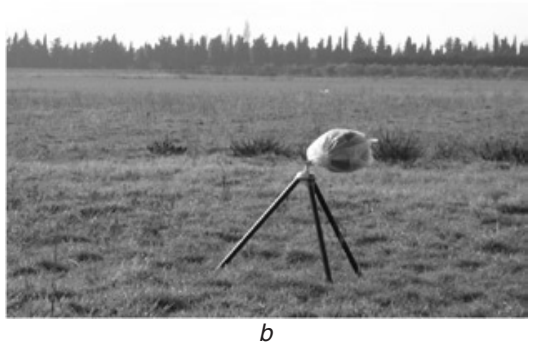

$b$

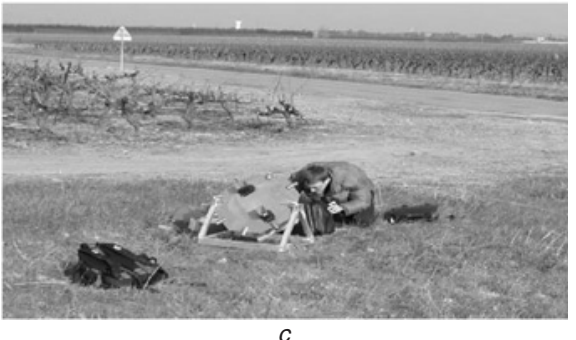

Fig. 5 Calibration devices

$a$ Trihedral corner reflector

$b$ Luneberg sphere

$c$ Transponder 


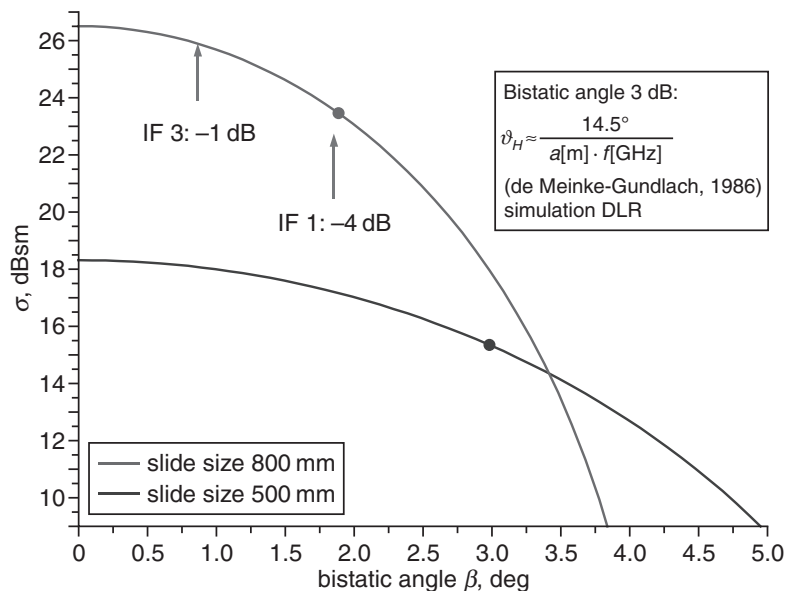

Fig. 6 Bistatic patterns of two trihedral corner reflectors The $3 \mathrm{~dB}$ pattern is less than $2^{\circ}$ wide for a $80 \mathrm{~cm}$ trihedral

For the calibration of bistatic images, we deployed a transponder which was originally designed to calibrate X-SAR, the German radar onboard the shuttle mission. The transponder response is wide enough to be used for the calibration of the bistatic images, shown in Fig. 7.

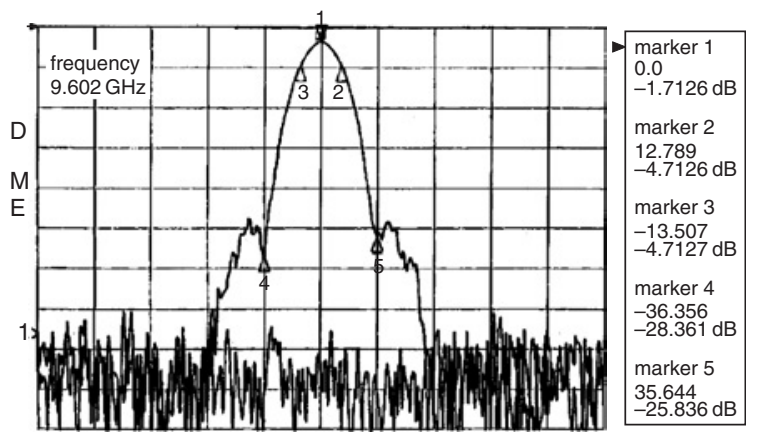

Fig. 7 Transponder response

This transponder was initially developed for X-SAR

\subsection{Description of the acquisitions}

Table 5 describes the different configurations flown during the campaign. The quasi-monostatic configurations were flown at two different altitudes, in order to study the effect of different signal to noise ratios and to have different interferometric baseline angles. Furthermore, the low altitude configurations allowed also for a recording of the air-to-air direct path radar signal which may be used for phase synchronisation $[14,17]$.

\section{Processing of bistatic images}

The processing of bistatic images was first done with the regular monostatic RAMSES SAR processor by using the average trajectory of the two planes as the 'monostatic' equivalent. This provided nice-looking images with good impulse responses for all configurations. However the resulting image geometry was extremely distorted for the ONERA configurations as the monostatic approximation is clearly not appropriate in this case. In order to analyse the influence of bistatic angle on scattering, it was necessary to develop a more complex processing taking into account the bistatic geometry and allowing a proper projection of the data into a common cartographic system. This processing is done in two steps. During the first step, the processing is based on the recorded trajectories and motions of the two planes with a processor adapted to the bistatic geometry:

In particular, integration time dependency to range is no more proportional and Doppler varies with range along a constant squint angle. Bistatic antenna pattern compensation is also implemented.

Clock drift between the radars must be evaluated and compensated for. Typical drifts observed during a one minute acquisition are a few $\mu \mathrm{s}$, with $1 \mu \mathrm{s}$ drift resulting in $150 \mathrm{~m}$ slant range error. Estimation of this clock drift is performed by comparing the bistatic and the monostatic image geometry as illustrated in Fig. 8. A bistatic image distortion model was developed which provides a library of functions (and their derivatives) for mapping image

Table 5: Description of the acquisitions

\begin{tabular}{|c|c|c|c|c|}
\hline \multirow[t]{2}{*}{ Acquisition identifier } & \multirow[t]{2}{*}{ Configuration } & \multicolumn{3}{|c|}{ Transmitter characteristics } \\
\hline & & Transmit radar & Bandwidth & Polarisation \\
\hline Bi101 & Quasi-mono & RAMSES & 50 & V \\
\hline Bi102 & Quasi-mono & RAMSES & 50 & V \\
\hline Bi103 & Quasi-mono & RAMSES & 50 & V \\
\hline $\mathrm{Bi} 104$ & Quasi-mono & RAMSES & 100 & V \\
\hline Bi105 & Quasi-mono & E-SAR & 100 & $\mathrm{~V}$ \\
\hline Bi108 & Steep & E-SAR & 100 & $\mathrm{~V}$ \\
\hline $\mathrm{Bi} 201$ & Grazing & E-SAR & 100 & V \\
\hline $\mathrm{Bi} 202$ & Grazing & E-SAR & 50 & V \\
\hline $\mathrm{Bi} 203$ & Steep & E-SAR & 50 & V \\
\hline $\mathrm{Bi} 204$ & Steep & E-SAR & 100 & $\mathrm{~V}$ \\
\hline Bi205 & Steep & RAMSES & 50 & $\mathrm{~V}$ \\
\hline $\mathrm{Bi} 206$ & Steep & RAMSES & 100 & $\mathrm{~V}$ \\
\hline $\mathrm{Bi} 207$ & Quasi-mono & E-SAR & 100 & $\mathrm{~V}$ \\
\hline $\mathrm{Bi} 208$ & Quasi-mono & E-SAR & 100 & V \\
\hline $\mathrm{Bi} 209$ & Quasi-mono & E-SAR & 100 & $\mathrm{~V}$ \\
\hline $\mathrm{Bi} 210$ & Quasi-mono & RAMSES & 100 & $\mathrm{~V}$ \\
\hline $\mathrm{Bi} 211$ & Quasi-mono & RAMSES & 50 & $\mathrm{~V}$ \\
\hline $\mathrm{Bi} 212$ & Quasi-mono & E-SAR & 50 & V \\
\hline
\end{tabular}




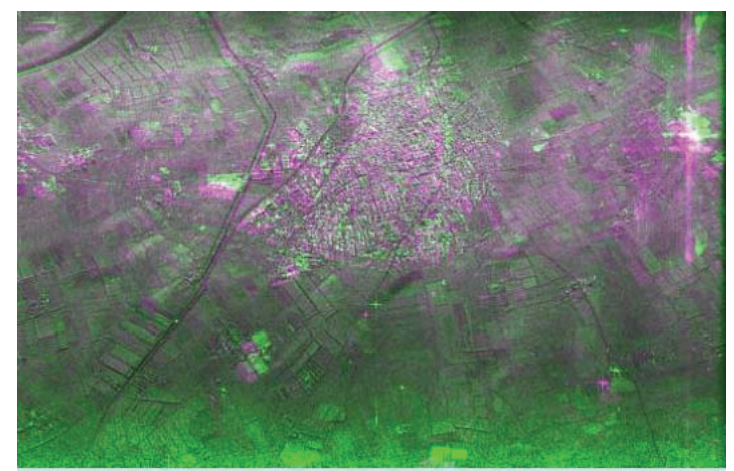

Fig. 8 Quasi-monostatic configuration, superposition of monostatic and bistatic images

The planes are flying from left to right at the top of the images. One can clearly detect the large shift (in both directions) between the two images. This shift is linked to the drift between the two clocks and can be computed by performing sub-image correlation along the image. Clock shift can then be inferred from this spatial shift

coordinates to geographical coordinates (and conversely) depending on trajectories, system parameters, and clock drift. Based on these functions, the observed distortion between the bistatic image (including drift effect) and the monostatic image (driftless) obtained through local image correlation can be translated into an estimated clock drift. Once the clock drift is known (Fig. 9), the bistatic image can be reprocessed.

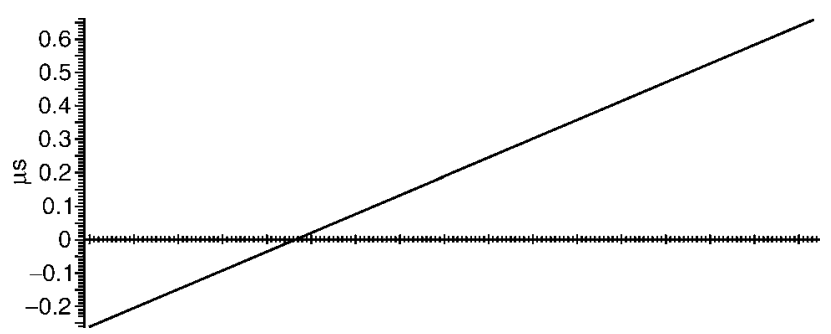

Fig. 9 We observe a clock drift along the acquisition linked to a slight difference in the STALO frequencies

This variation is of the order of $1 \mu \mathrm{s}$ for about 1 minute. There is a strong linear component dominating an underlying variation
The bistatic and monostatic images are now superposed with an accuracy of better than 1 pixel. This is adequate for most applications. However, for interplatform interferometry, the superposition accuracy must be of the order of $1 / 10$ of a pixel. In order to meet this requirement, another step in the processing has to be performed. A map of sub-pixel shifts and an interferogram are computed based on local maximisation of interferometric coherence. The interferometric phase behaviour, the residual fringes and the variation of subpixel shifts in the image are used as an input to fine tune the clock drift (order of a few ns) and the bistatic trajectory using the distortion model described earlier. The output of this process is a better knowledge of the relative trajectory of the two planes. This step has been detailed in [21]. Fig. 10 is a bistatic cross-platform interferometric image where the interferometric phase is coded as a colour and the amplitude as the intensity.

\section{Calibration of the bistatic images}

The calibration of the quasi-monostatic bistatic images is simply done by using the passive calibration targets (trihedral and Luneberg sphere deployed on the scene).

Calibration of the ONERA configuration bistatic images is more of a challenge as the passive targets can no longer be used as the bistatic angle is of the order of $20^{\circ}$. Fig. 6 provides the bistatic behaviour of a trihedral corner reflector for two different sizes. As can be seen in this Figure, the width of the trihedral pattern is extremely narrow (a few degrees) and therefore cannot be used for bistatic angles of the order of $20^{\circ}$.

The calibration of a bistatic image is performed following the synoptic presented in Fig. 11:

- The corresponding monostatic image is calibrated using the trihedral corner reflector response. Note that we always acquired a monostatic image at the same time as a bistatic image (both systems were receiving). From this calibrated monostatic image, the gain of the transponder was determined (taking into account the off-boresight angle). This is essential as the transponder was originally designed for X-SAR and therefore had a very high gain, potentially saturating the airborne data. In order to avoid

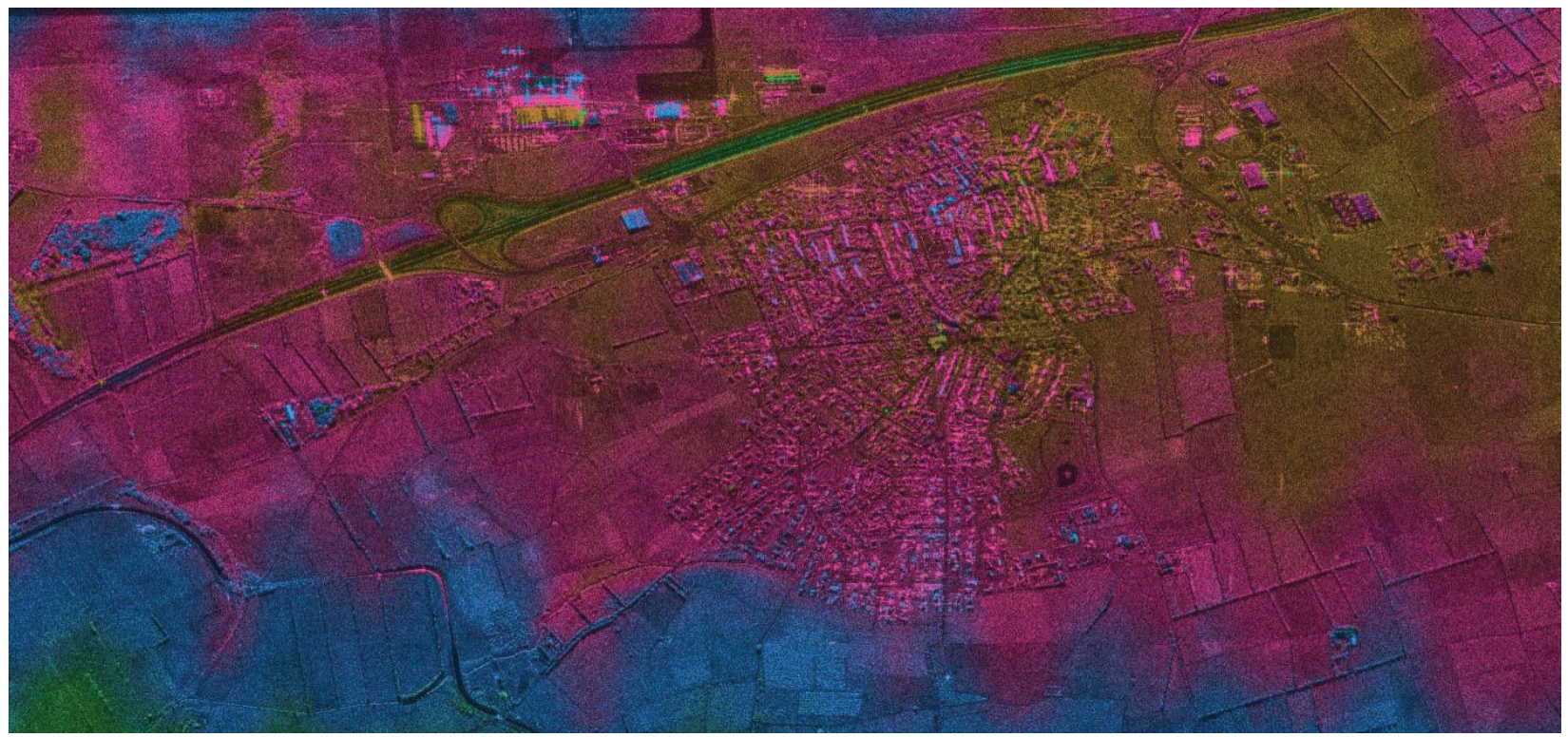

Fig. 10 Bistatic interferometric image: the quasi-monostatic image coloured with the cross-platform interferometric phase

One can notice buildings (in a blue shade) associated with a higher elevation and the freeway in the top part of the image characterised by a lower altitude as it is buried 


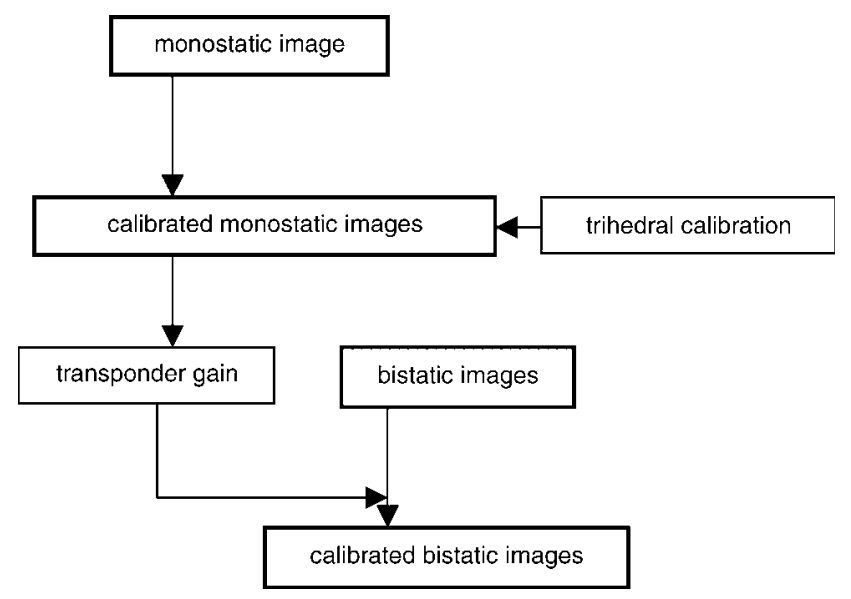

Fig. 11 Synoptic of the calibration procedure for the bistatic images

this problem, its gain was lowered using an uncalibrated built-in attenuator resulting in an unknown gain.

- The expected level of the transponder in the bistatic image is then computed taking into account both offboresight angles and an overall calibration factor for the bistatic image is then obtained.

The calibration procedure is validated over the quasimonostatic images where we can use either the transponder or the trihedral methods.

\section{Image analysis}

\subsection{Quasi-monostatic against monostatic}

It is interesting to note that the quasi-monostatic and the monostatic images present noticeable differences. As expected, these differences are observed in the urban areas, where scattering is dominated by dihedral effects known to be extremely sensitive to aspect angle, especially when the dihedral facets are large. In addition, significant changes are also visible over agricultural fields. This is rather unexpected since natural surfaces are supposed to have a slowly varying scattering pattern for which a $0.1^{\circ}$ change should have no effect. This will require further analysis.

In Fig. 12, a colour composite image of the monostatic and quasi-monostatic images is presented. The differences in shades between different fields are clearly visible.

\subsection{Bistatic angle effect on the scattering}

This colour composite image is the superposition of three bistatic images acquired with three bistatic angles. It illustrates the effect of bistatic angle on scattering. The blue colour corresponds to an almost monostatic image acquired in the DLR configuration. In the monostatic case, the double bounce reflection is very strong in the village. The green image corresponds to a bistatic angle further away from the specular direction and the denser vegetation is green indicating that for vegetation, the scattering is very homogeneously distributed over all bistatic directions.

The stereoscopic effect that can be observed along the tree-edges in Fig. 13 is linked to the different shadowing effect from the tree-edges associated with the different observation geometry. As expected, the shadow increases with incidence angle as can be observed when comparing the monostatic image and the grazing image. However, the shadows seem better defined and wider in the steep
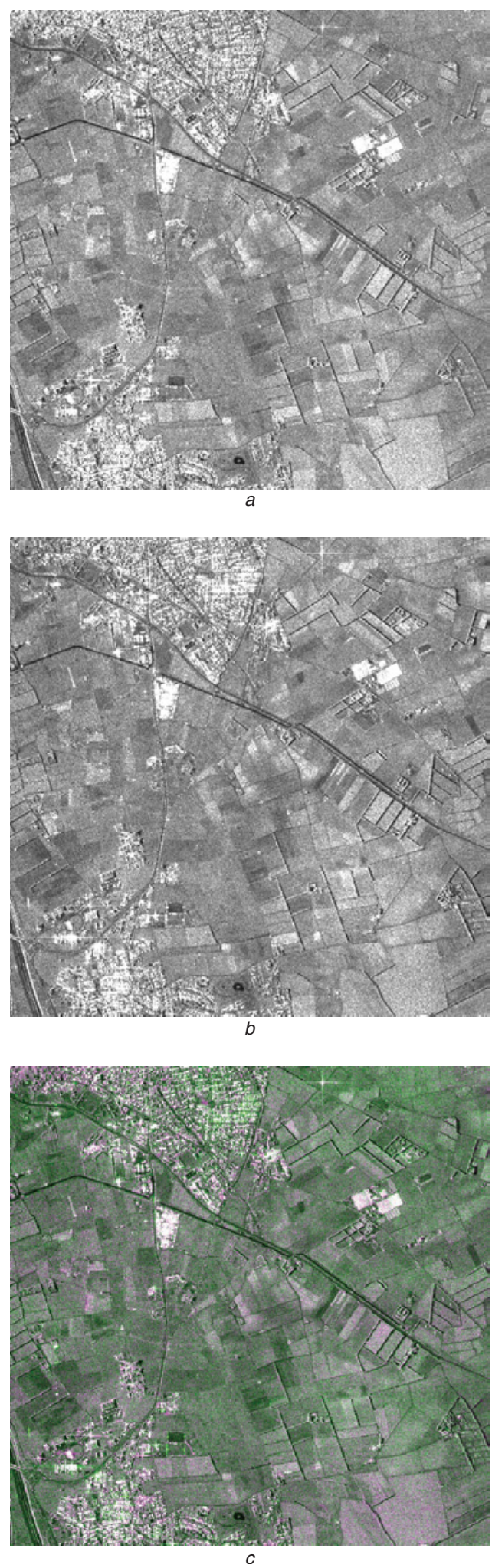

Fig. 12 Comparison between the monostatic and the quasimonostatic bistatic image

$a$ Monostatic image

$b$ Quasi-monostatic image

$c$ Colour composite of the two images

configuration than in the monostatic case. This is linked to the overlay phenomena. In ground geometry, the distance between the top of the tree edge and the shadow limit is larger for the steep configuration case:

- The shadow limit is at the same ground position for both images as it is determined by the larger incidence angle (which is $55^{\circ}$ in both cases). 

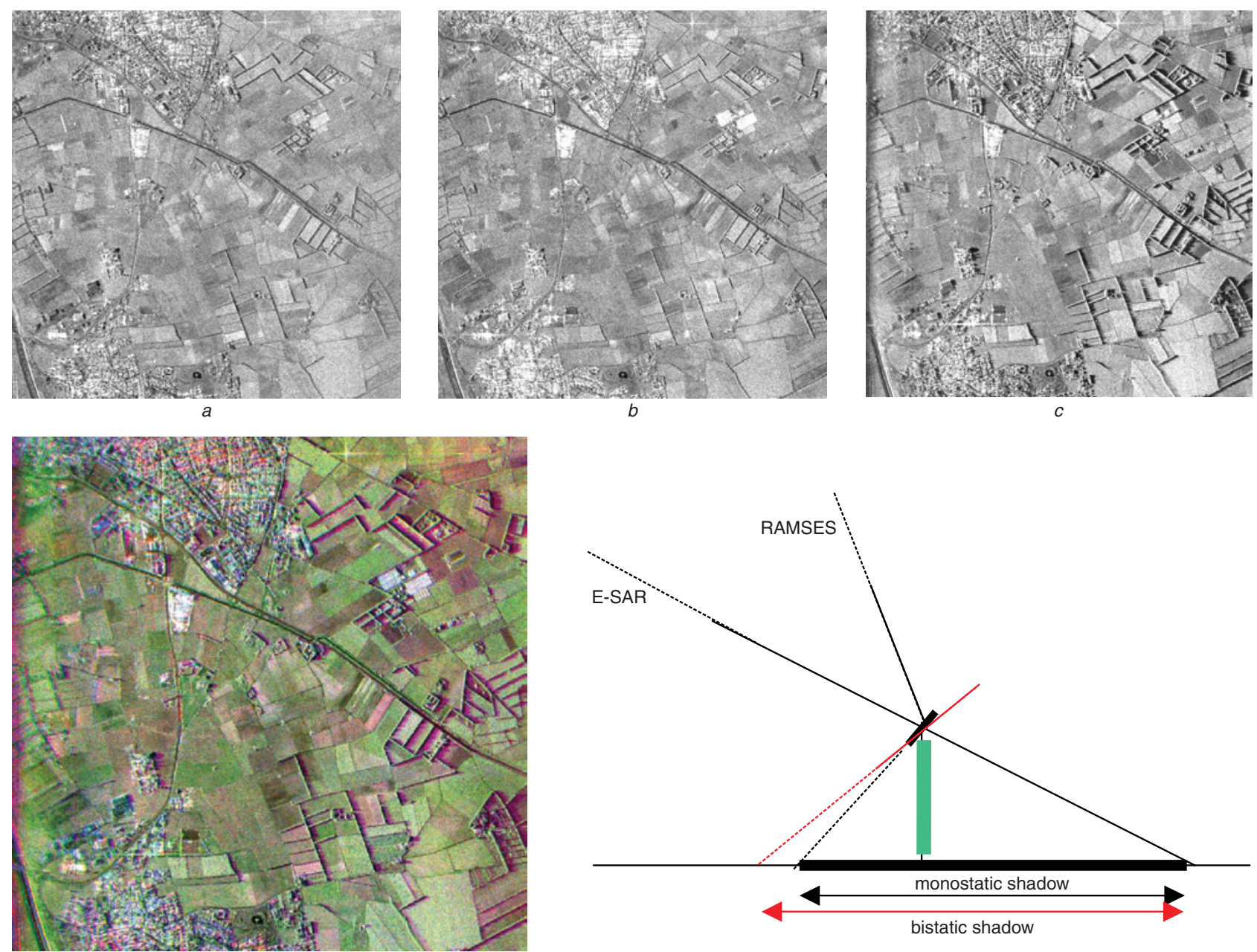

Fig. 13 Comparison between the different bistatic images

$a$ Steep angle

$b$ Quasi-monostatic

$c$ Grazing angle configuration

The colour composition was obtained by coding $a$ in red, $b$ in blue and $c$ in green

In the schematic the shadow length is illustrated for the monostatic and the bistatic steep angle configuration. The shadow in the bistatic steep angle configuration is shown to be longer, as observed in the data

- The hedge top position is distorted owing to an overlay phenomenon. Its position, as projected on the ground will be further away from the real position in the bistatic case as the iso-distance surface (an ellipsoid) for the bistatic case has a smaller slope than the corresponding iso-distance sphere associated with the quasi-monostatic case as described in Fig. 13.

Fig. 13 is a comparison between the quasi-monostatic image and the steep configuration image. Preliminary analysis was conducted on the different surfaces and clearly shows that the bistatic angle helps characterise natural surfaces and discriminate between different types of land cover (vineyard, orchard, wheat, bare fields ...).

\section{Conclusion}

These first results from the ONERA/DLR bistatic SAR campaign have already proved the great potential of bistatic SAR imaging for signature analysis, urban area remote sensing (less saturation owing to the absence of strong dihedral reflections), and natural surface characterisation. Furthermore, the cross-platform interferometry, illustrated here, clearly demonstrates that this technique can be used for terrain elevation retrieval from only one transmitting and two receiving radars. This supports the set-up of spaceborne multistatic SAR systems such as the interferometric cartwheel, pendulum or tandem missions. Note that the orbital fringe removal is expected to be much simpler than in the above described airborne experiment since the typical satellite trajectories are usually orders of magnitude smoother than the aircraft ones.

The preliminary analysis of the images is extremely encouraging even though a lot remains to be done. The data processing has to be improved and the phenomenology has to be studied more in depth. These topics will be investigated in the near future.

\section{Acknowledgments}

The authors would like to thank the ONERA/RAMSES team, the DLR/E-SAR team for their help and support, namely Olivier Ruault du Plessis, Daniel Heuzé, Patrick Fromage, Jack Peret, Jean-luc Peron, Jean-Paul Canny, Colette Coulombeix, Xavier Dupuis, Philippe Martineau from ONERA and Bernd Gabler, Wolfgang Schneider, Franz Witte, Hans-Jürgen Müller, and Mark Rodriguez Cassola for DLR. The authors would also like to express our gratitude both to the CEV pilots and navigators, François Duflot, Didier Ilcilnkas, Benoit Smith, Hubert Greuet, Laurence Lambert and the DLR pilots Klaus Dietl and Stefan Grillenbeck for bringing together their 
competence to help solve the critical flight accuracy requirements. RAMSES was developed and is currently upgraded through funding from the DGA. RAMSES bistatic flights were made possible by DGA/STTC providing the necessary clearance.

\section{References}

1 Willis, N.J.: 'Bistatic radar' (Artech House, 1991)

2 Moccia, A., Chiacchio, N., and Capone, A.: 'Spaceborne bistatic synthetic aperture radar for remote sensing applications', Int. J. Remote Sens., 2000, 21, (18), pp. 3395-3414

3 Krieger, G., Fiedler, H., and Moreira, A.: 'Bi- and multi-static SAR: potential and challenges'. Proc. EUSAR 2004, Ulm, Germany, 25-27 May 2004

4 Evans, N.B., Lee, P., and Girard, R.: 'The RADARSAT-2/3 topographic mission'. Proc. EUSAR 2002, Cologne, Germany, pp. 37-39

5 Massonnet, D.: 'The interferometric cartwheel, a constellation of low cost receiving satellites to produce radar images that can be coherently combined', Int. J. Remote Sens., 2001, 22, (12), pp. 2413-2430

6 Krieger, G., Fiedler, H., Mittermayer, J., Papathanassiou, K., and Moreira, A.: 'Analysis of multistatic configurations for spaceborne SAR interferometry', IEE Proc., Radar Sonar Navig., 2003, 150, (3), pp. 87-96

7 Zebker, H., Farr, T., Salazar, R., and Dixon, T.: 'Mapping the world's topography using radar interferometry: the TOPSAT mission', Proc. IEEE, 1994, 82, (12), pp. 1774-1786

8 Hanssen, R.: 'Radar interferometry: data interpretation and error analysis' (Kluwer Academic Publishers, Dordrecht, 2001)

9 Farr, T., and Kobrick, M.: 'The shuttle radar topography mission'. Proc. EUSAR 2004, Ulm, Germany, 25-27 May 2004

10 Hartl, P., and Braun, H.M.: 'Bistatic radar in space', in 'Space based radar handbook’ (Artech House, 1989)
11 Yates, G., Horne, M., Blake, A., Middleton, R., and Andre, D.B.: 'Bistatic image formation'. Proc. EUSAR 2004, Ulm, Germany, 25-27 May 2004

12 Ender, J., Waleterscheid, I., and Brenner, A.: 'New aspects of bistatic SAR: processing and experiments'. Proc. IGARSS 04, Anchorage, USA, 20-24 Sept. 2004

13 Dubois-Fernandez, P., Cantalloube, H., Ruault du Plessis, O., Wendler, M., Horn, R., Vaizan, B., Boumahmoud, A., Coulombeix, C., Heuzé, D., Krieger, G., and Gabler, B.: 'ONERA-DLR bistatic SAR experiment: design of the experiment and preliminary results'. Proc. ASAR Workshop 2003, CEOS WGCV SAR, Montréal, Canada, 25-27 June 2003

14 Wendler, M., Krieger, G., Horn, R., and Gabler, B.: 'Results of a bistatic airborne SAR experiment'. Proc. Int. Radar Symp., IRS 2003, Dresden, Germany, 30 Sept.-2 Oct. 2003, pp. 247-253

15 Dubois-Fernandez, P., Ruault du Plessis, O., et al.: 'The RAMSES experimental SAR system'. Proc. IGARSS'02, Toronto, Canada, July 2002

16 Horn, R., Moreira, A., Buckreuss, S., and Scheiber, R.: 'Recent developments of the airborne SAR system E-SAR of DLR'. Proc. EUSAR 2000, Munich, Germany, 2000

17 Krieger, G., Wendler, M., Fiedler, F., and Werner, M.: 'DLR technical note on the preparation of a bistatic airborne SAR experiment DLR/ ONERA: interferometric X-band configurations with along-track separation', Feb. 2002

18 Vaizan, B., Dubois-Fernandez, P., and du Plessis, O.: 'ONERA status report bistatic experiment', April 2002

19 Auterman, J.L.: 'Phase stability requirements for a bistatic SAR'. Proc. IEEE Nat. Radar Conf., Atlanta, USA, pp. 48-52

20 Weiss, M.: 'Time and frequency synchronisation aspects for bistatic SAR systems'. Proc. EUSAR 2004, Ulm, Germany, 25-27 May 2004

21 Cantalloube, H., Dubois-Fernandez, P., and Giroux, V.: 'Challenges in SAR processing for airborne bistatic acquisitions'. Proc. EUSAR 2004, Ulm, Germany, 25-27 May 2004 